\title{
Thermodynamics of Modified Chaplygin Gas and Tachyonic Field
}

\author{
Samarpita Bhattacharya* and Ujjal Debnath ${ }^{\dagger}$ \\ Department of Mathematics, Bengal Engineering and Science University, Shibpur, Howrah-711 103, India.
}

(Dated: July 7, 2021)

\begin{abstract}
Here we generalize the results of the work of ref. [10] in modified Chaplygin gas model and tachyonic field model. Here we have studied the thermodynamical behaviour and the equation of state in terms of volume and temperature for both models. We have used the solution and the corresponding equation of state of our previous work [12] for tachyonic field model. We have also studied the thermodynamical stability using thermal equation of state for the tachyonic field model and have shown that there is no critical points during thermodynamical expansion. The determination of $T_{*}$ due to expansion for the tachyonic field have been discussed by assuming some initial conditions. Here, the thermal quantities have been investigated using some reduced parameters.

PACS numbers:
\end{abstract}

\section{INTRODUCTION}

Data $[1,2]$ collecting from type Ia Supernovae explosion suggest that a new kind of matter with positive energy density and with negative pressure is one of the main candidate behind the acceleration of the present day universe. This new kind of matter is commonly known as Q-matter or dark energy and very recently WMAP data [3] have revealed that Q-matter possesses $70 \%$ of the universe. Cosmologists suggest many candidates for the dark energy. Among these the first one is cosmological constant $\Lambda$ [4]. But due to low energy scale than the normal scale for constant $\Lambda$, the dynamical $\Lambda$ was introduced which is known as quintessence [5]. Again at very early stage of universe the energy scale for varying $\Lambda$ is not sufficient. So to avoid this problem, known as cosmic coincidence [6], a new field, called tracker field [7] was prescribed. In similar way there are many models [8] in Einstein gravity to best fit the data. At the present epoch, a lot of works has been done to solve this quintessence problem and most popular candidates for Q-matter has so far been a scalar field having a potential which generates a sufficient negative pressure. Various dark energy candidates have been proposed such as quintessence mentioned, k-essence, tachyon, phantom, Chaplygin gas, holographic dark energy etc [9].

Here we generalize the results of the work of Myung [10] in modified Chaplygin gas model [11] and tachyonic field model [12]. Here we have studied the thermodynamical behaviour and the equation of state in terms of volume and temperature for both models. We have used the solution and the corresponding equation of state of our previous work [12] for tachyonic field model. Recently Santos et al [13] have studied the thermodynamical stability in generalized and modified Chaplygin gas model. We'll study the thermodynamical stability [13] using adiabatic and thermal equation of states for the tachyonic field model [12] and investigate the behaviour of critical points during thermodynamical expansion.

\section{STUDY OF THERMODYNAMICS}

The Einstein field equations for homogeneous, isotropic and flat FRW universe are given by

$$
H^{2}=\frac{1}{3} \rho
$$

and

$$
\dot{H}=-\frac{1}{2}(\rho+p)
$$

\footnotetext{
* samarpita_sarbajna@yahoo.co.in

† ujjaldebnath@yahoo.com , ujjal@iucaa.ernet.in
} 
where $H\left(=\frac{\dot{a}}{a}\right)$ is the Hubble parameter. The continuity equation is given by

$$
\dot{\rho}+3 H(\rho+p)=0
$$

where $p$ is the isotropic pressure and $\rho$ is the energy density of the fluid defined by

$$
\rho=\frac{U}{V}
$$

Here, $U$ is the internal energy and $V$ is the volume of the universe.

We apply the combination of first law and second law of thermodynamics to the system with volume $V$. Then it leads to $[10]$

$$
T d S=d(\rho V)+p d V=d((\rho+p) V)-V d p
$$

The integrability condition of thermodynamic system is given by

$$
\frac{\partial^{2} S}{\partial T \partial V}=\frac{\partial^{2} S}{\partial V \partial T}
$$

which leads to the relation between pressure, energy density and temperature as

$$
d p=\frac{\rho+p}{T} d T
$$

From (5) and (7), we get

$$
d S=d\left(\frac{(\rho+p) V}{T}\right)
$$

Therefore integrating, we get the expression of the entropy as

$$
S=\frac{(\rho+p) V}{T}
$$

or, the temperature can be written as

$$
T=\frac{(\rho+p) V}{S}
$$

In the next two sections, we consider two dark energy models: (i) Modified Chaplygin gas and (ii) Tachyonic field and we study the thermodynamical behaviours for these models.

\section{MODEL I : MODIFIED CHAPLYGIN GAS}

We start with an exotic fluid, named Modified Chaplygin Gas which obeys the following adiabatic equation of state [11]

$$
p=A \rho-\frac{B}{\rho^{\alpha}}
$$


and $A>0, B>0$ and $0 \leq \alpha \leq 1$.

Solving the equations (3) and (11), we get the expression of the density as

$$
\rho(a)=\left[\frac{B}{1+A}+\frac{C}{a^{3(1+A)(1+\alpha}}\right]^{\frac{1}{1+\alpha}}
$$

where $C(>0)$ is a constant of integration.

Since $V$ is the volume, so we have $V=a^{3}$ and from equations (12) and (11), we get the expressions of the density and the pressure in terms of volume $V$ as

$$
\rho(V)=\left(\frac{B}{1+A}+\frac{C}{V^{(1+A)(1+\alpha)}}\right)^{\frac{1}{1+\alpha}}
$$

and

$$
p(V)=\frac{\frac{A C}{V^{(1+\alpha)(1+A)}}-\frac{B}{1+A}}{\left(\frac{B}{1+A}+\frac{C}{V^{(1+\alpha)(1+A)}}\right)^{\frac{\alpha}{1+\alpha}}}
$$

Furthermore, the equation of the state $w(V)$ for modified Chaplygin gas is given by

$$
w(V)=\frac{p}{\rho}=\frac{\frac{A C}{V^{(1+\alpha)(1+A)}-\frac{B}{1+A}}}{\frac{B}{1+A}+\frac{C}{V^{(1+\alpha)(1+A)}}}
$$

and the square speed of sound is given by

$$
v^{2} \equiv \frac{\partial p}{\partial \rho}=A-\frac{B}{\alpha}\left(\frac{B}{1+A}+\frac{C}{V^{(1+\alpha)(1+A}}\right)^{\frac{1-\alpha}{1+\alpha}}
$$

and consequently, considering equations (10), (13) and (14) we get the expression of temperature as

$$
T(V)=\frac{(1+A) C}{S V^{A+(1+A) \alpha}}\left(\frac{B}{1+A}+\frac{C}{V^{(1+\alpha)(1+A)}}\right)^{-\frac{\alpha}{1+\alpha}}
$$

Since volume $V$ lies between 0 and $\infty$. So we see that the temperature $T \rightarrow \infty$ as $V \rightarrow 0$ and $T \rightarrow 0$ as $V \rightarrow \infty$, so the third law of thermodynamics is satisfied for modified Chaplygin gas model.

From the integrability condition and using (7) and (11), we have

$$
\frac{A \rho^{1+\alpha}+B \alpha}{(A+1) \rho^{2+\alpha}-B \rho} d \rho=\frac{d T}{T}
$$

Solving we get,

$$
\left(\rho^{1+\alpha}-\frac{B}{1+A}\right)^{\frac{A+\alpha(1+A)}{(1+A)(1+\alpha)}} \rho^{-\alpha}=\frac{T}{T_{*}}\left(\frac{B}{A}\right)^{\frac{A}{(1+A)(1+\alpha)}}\left(\frac{1}{1+A}\right)^{\frac{A+\alpha(1+A)}{(1+A)(1+\alpha)}}
$$

which is a relation between $\rho$ and $T$ and $T_{*}$ is the integration constant.

Again, using $w=\frac{p}{\rho}$ and (11), the equation (19) gives 


$$
\left(\frac{B}{A-w}-\frac{B}{1+A}\right)^{\frac{A+\alpha(1+A)}{(1+A)(1+\alpha)}}\left(\frac{B}{A-w}\right)^{-\frac{\alpha}{1+\alpha}}=\frac{T}{T_{*}}\left(\frac{B}{A}\right)^{\frac{A}{(1+A)(1+\alpha)}}\left(\frac{1}{1+A}\right)^{\frac{A+\alpha(1+A)}{(1+A)(1+\alpha)}}
$$

which is a relation between $w$ and $T$ provided $w \neq A$.

Finally the heat capacity can be calculated in terms of $V$ and $T$ Tas

$$
C_{V}(T)=V \frac{\partial \rho}{\partial T}=\frac{V}{T \rho}\left(\frac{A \rho^{1+\alpha}+B \alpha}{(A+1) \rho^{1+\alpha}-B}\right)=\frac{V}{T}\left(\frac{A-w}{B}\right)^{\frac{1}{1+\alpha}}\left(\frac{(1+\alpha) A-\alpha w}{1+w}\right)
$$

where, $w$ is related with temperature $T$ governed by the equation (20).

For simplicity, we take $\alpha=1$ in the equation of state (11), i.e.,

$$
p=A \rho-\frac{B}{\rho}
$$

Solving the quadratic equation in $\rho$ and inserting the value in (7) we get a quadratic equation of $p$ which is

$$
(1+A) p^{2}-\frac{S T}{V}(1+2 A) p+\left(\frac{A S^{2} T^{2}}{V^{2}}-B\right)=0
$$

Solving the above equation and considering only the negative sign, we obtain

$$
p V=\frac{S T(1+2 A)-\sqrt{S^{2} T^{2}+4 B(1+A) V^{2}}}{2(1+A)}
$$

For adiabatic process, we know that the entropy $S=$ constant. So in the case, taking $S=2$, $(24)$ gives the simpler form

$$
p V=\frac{T(1+2 A)-\sqrt{T^{2}+B(1+A) V^{2}}}{(1+A)}
$$

On the other hand, we have the quadratic equation for energy density $\rho$ (taking $\alpha=1)$

$$
(1+A) V \rho^{2}-S T \rho-B V=0
$$

whose solution leads to

$$
\rho V=\frac{S T}{2(1+A)}+\frac{1}{2(1+A)} \sqrt{S^{2} T^{2}+4 B(1+A) V^{2}}
$$

For the case $S=2$, it also reduces to the simpler form

$$
\rho V=\frac{1}{1+A}\left(T+\sqrt{T^{2}+B(1+A) V^{2}}\right)
$$

Now dividing (24) by (27) leads to the equation of state for modified Chaplygin gas as functions of $T$ and $V$ :

$$
\omega(T, V)=A-\frac{B}{\rho^{2}(T, V)}
$$

Now from equations (24) and (27), we get

(i) $\rho \rightarrow \frac{S T}{(1+A) V} \approx \infty$ and $p \rightarrow \frac{A S T}{(1+A) V}=A \rho \approx \infty$ as $V \rightarrow 0$ provided $A \neq 0$.

(ii) $\rho \rightarrow \sqrt{\frac{B}{1+A}}$ and $p \rightarrow-\sqrt{\frac{B}{1+A}}=-\rho$ as $V \rightarrow \infty$ i.e., the fluid becomes a cosmological constant. 


\section{MODEL II : TACHYONIC FIELD}

The energy density $\rho$ and the pressure $p$ of the tachyonic field are [12],

$$
\rho=\frac{V(\phi)}{\sqrt{1-\dot{\phi}^{2}}}
$$

and

$$
p=-V(\phi) \sqrt{1-\dot{\phi}^{2}}
$$

where $\phi$ is the tachyonic field and $V(\phi)$ is the corresponding potential. Now take a simple form of $V=$ $\left(1-\dot{\phi}^{2}\right)^{-m},(m>0)$ as described in the ref. [12], so the solution of $V$ becomes

$$
V=\left[1+\left(\frac{V^{*}}{a^{3}}\right)^{\frac{2}{1+2 m}}\right]^{m}
$$

where $V^{*}$ is a positive constant. Now using equations (1), (2) and (30-32), we obtain the adiabatic equation of the state for the tachyonic field (due to this particular solution) as

$$
p=-\rho^{\frac{2 m-1}{2 m+1}}
$$

We derive the energy density equation from (30) as

$$
\rho(a)=\left[1+\left(\frac{V^{*}}{a^{3}}\right)^{\frac{2}{1+2 m}}\right]^{\frac{2 m+1}{2}}
$$

\section{A. Adiabatic equation of state for tachyonic field}

Now from equations (33) and (34), we obtain the energy density and pressure in terms of volume $V$ as

$$
\rho=\left[1+\left(\frac{V^{*}}{V}\right)^{\frac{2}{2 m+1}}\right]^{\frac{2 m+1}{2}}
$$

and

$$
p=-\left[1+\left(\frac{V^{*}}{V}\right)^{\frac{2}{2 m+1}}\right]^{\frac{2 m-1}{2}}
$$

For small $V$, we find $\rho \approx \frac{V^{*}}{V}$ and $p \approx-\left(\frac{V^{*}}{V}\right)^{\frac{2 m-1}{2 m+1}}$ which is negatively very large if $m>\frac{1}{2}$ and nearly equal to 0 if $m<\frac{1}{2}$. Also for large $V$, we see that $\rho \approx 1+\frac{2 m+1}{2}\left(\frac{V^{*}}{V}\right)^{\frac{2}{2 m+1}}$ and $p \approx-1-\frac{2 m-1}{2}\left(\frac{V^{*}}{V}\right)^{\frac{2}{2 m-1}}$.

Furthermore the equation of state $w(V)=p / \rho$ and square speed of sound $v^{2}(V)$ are given by

$$
w(V)=-\frac{1}{1+\left(\frac{V^{*}}{V}\right)^{\frac{2}{2 m+1}}}
$$


and

$$
v^{2}(V)=-\left(\frac{2 m-1}{2 m+1}\right) \frac{1}{1+\left(\frac{V^{*}}{V}\right)^{\frac{2}{2 m+1}}}=\left(\frac{2 m-1}{2 m+1}\right) w(V)
$$

From Equation (36), we have

$$
\left(\frac{\partial p}{\partial V}\right)_{S}=-\left(\frac{2 m-1}{2 m+1}\right) \frac{p}{V}\left(\frac{V^{*}}{V}\right)^{\frac{2}{2 m+1}}
$$

Since Tachyonic field propagated between dust and $\Lambda$ CDM, so pressure $p$ must be negative and $m$ must be satisfied $0<m<\frac{1}{2}$. Therefore from (39) we must have [14]

$$
\left(\frac{\partial p}{\partial V}\right)_{S}<0
$$

i.e., pressure is reduced through the adiabatic expansion. So the tachyonic fluid along its evolution is thermodynamically stable. But this condition is not enough for stability of thermodynamics. It is also necessary to determine if the pressure reduces or remains constant as the fluid expands at constant temperature $T$, in the same region where eq. (39) is negative [14]. Thus, one must also verify if

$$
\left(\frac{\partial p}{\partial V}\right)_{T} \leq 0
$$

and the thermal capacity of the constant volume [32],

$$
C_{V}>0
$$

then the tachyonic fluid along its evolution is fully thermodynamically stable. These will be discussed in the following subsection.

\section{B. Thermal equation of state for tachyonic field}

The equation of state for the tachyonic field is considered in equation (33) and the energy density for tachyonic field is considered in equation (4). From general thermodynamics, we write $[13,14]$

$$
\left(\frac{\partial U}{\partial V}\right)_{S}=-p
$$

From (4), (33) and (43), we obtain

$$
\left(\frac{\partial U}{\partial V}\right)_{S}=\left(\frac{U}{V}\right)^{\frac{2 m-1}{2 m+1}}
$$

whose solution is given by

$$
U=V\left[1+\left(\frac{V^{*}}{V}\right)^{\frac{2}{2 m+1}}\right]^{\frac{2 m+1}{2}}
$$

Here, $V^{*}$ may be considered as a function of entropy $S$ or a universal constant. From general thermodynamics [14], the temperature $T$ of the tachyonic field can be determined from the following equation: 


$$
T=\left(\frac{\partial U}{\partial S}\right)_{V}=\left(V^{*}\right)^{\frac{1-2 m}{1+2 m}}\left[V^{\frac{2}{2 m+1}}+\left(V^{*}\right)^{\frac{2}{2 m+1}}\right]^{\frac{2 m-1}{2}} \frac{d V^{*}}{d S}
$$

If $V^{*}$ is chosen as a universal constant then $\frac{d V^{*}}{d S}=0$. In such conditions, the temperature will be zero for any value of the volume or pressure of the gas. Thus, the isotherm $T=0$ is simultaneously an isentropic curve (adiabatic) at $S=$ constant and this violates the third law of thermodynamics. Therefore, to discuss extensively the thermodynamic stability of the tachyonic field, it is necessary to assume that the condition is not satisfied.

If $V \gg V^{*}$, that is $\mathrm{V}$ is very large, then (46) reduces to

$$
T \approx\left(\frac{V}{V^{*}}\right)^{\frac{2 m-1}{2 m+1}} \frac{d V^{*}}{d S}
$$

Since $T>0$ for adiabatic expansion, so from (47), we must have $\frac{d V^{*}}{d S}>0$. Now from (45), we see that the dimension of $V^{*}$ is same as the dimension of $U$. So, we may write $V^{*}$ as [13] $V^{*}=T_{*} S$, where $T_{*}$ is some constant related to the temperature. So $T_{*}=\frac{d V^{*}}{d S}$. Now, from (46), we obtain,

$$
T=T_{*}^{\frac{2}{2 m+1}} S^{\frac{1-2 m}{1+2 m}}\left[V^{\frac{2}{2 m+1}}+T_{*}^{\frac{2}{2 m+1}} S^{\frac{2}{1+2 m}}\right]^{\frac{2 m-1}{2}}
$$

By solving (48), we obtain the expression of entropy $S$ as

$$
S=\frac{V}{T_{*}}\left[\left(\frac{T}{T_{*}}\right)^{\frac{2}{2 m-1}}-1\right]^{-\frac{2 m+1}{2}}
$$

Since in our tachyonic field model, $m<\frac{1}{2}$ and entropy $S$ is always positive. So from above result, we can conclude that $0<T<T_{*}$.

From equations (7) and (33), we get

$$
\frac{d p}{p-(-p)^{\frac{2 m+1}{2 m-1}}}=\frac{d T}{T}
$$

and after integration we obtain the expression pressure in terms of temperature as

$$
p=-\left[1-\left(\frac{T}{T_{*}}\right)^{\frac{2}{1-2 m}}\right]^{\frac{1-2 m}{2}}
$$

and using eq. (33), we obtain the expression for density as

$$
\rho=\left[1-\left(\frac{T}{T_{*}}\right)^{\frac{2}{1-2 m}}\right]^{-\frac{2 m+1}{2}}
$$

and hence the equation of state is obtained as

$$
w(T)=\frac{p}{\rho}=-1+\left(\frac{T}{T_{*}}\right)^{\frac{2}{1-2 m}}
$$


From the above expression, we see that $\rho \rightarrow 1$ and $p \rightarrow-1$ as $T \rightarrow 0$ and $\rho \rightarrow \infty$ and $p \rightarrow 0$ as $T \rightarrow T_{*}$ for $m<\frac{1}{2}$ which is expected in our model. So $T_{*}$ defines the temperature of dust and zero temperature occurs at $\Lambda \mathrm{CDM}$ stage.. We also see that, $p$ depends only on $T$ for any volume $V$. So we have $\left(\frac{\partial p}{\partial V}\right)_{T}=0$. Thus the second condition of the stability of thermodynamics is always satisfied. It is also interesting that the all derivatives of $p$ with respect to $T$ become zero for any volume $V$. Thus there is no critical points during the thermodynamical expansion.

Now the thermal capacity of the constant volume [14] can be calculated as

$$
C_{V}=T\left(\frac{\partial S}{\partial T}\right)_{V}=-\frac{2 m+1}{2 m-1} \frac{V}{T_{*}}\left(\frac{T}{T_{*}}\right)^{\frac{2}{2 m-1}}\left[\left(\frac{T}{T_{*}}\right)^{\frac{2}{2 m-1}}-1\right]^{-\frac{2 m+3}{2}}
$$

Since $0<m<\frac{1}{2}$ and $0<T<T_{*}$, so (54) shows that $C_{V}>0$ in the tachyonic field model. So the third condition of stability is always satisfied. So we may conclude that tachyonic field has a thermodynamically stable behaviour throughout the expansion.

\section{Determination of temperature}

Now we have to determine the temperature $T_{*}$ due to expansion for the tachyonic field. For this this purpose, let us assume the initial conditions: $\rho=\rho_{0}, p=p_{0}, V=V_{0}$ and $T=T_{0}$. So from equations (33) and (35), we obtain

$$
V^{*}=V_{0}\left(\rho_{0}^{\frac{2}{2 m+1}}-1\right)^{\frac{2 m+1}{2}}
$$

and

$$
p_{0}=-\rho_{0}^{\frac{2 m-1}{2 m+1}}
$$

Equations (35), (36) and (55), we obtain the energy density $\rho$ and pressure $p$ as a function of the volume $V$ :

$$
\rho=\left[1+\left(\rho_{0}^{\frac{2}{2 m+1}}-1\right)\left(\frac{V_{0}}{V}\right)^{\frac{2}{2 m+1}}\right]^{\frac{2 m+1}{2}}
$$

and

$$
p=-\left[1+\left(\rho_{0}^{\frac{2}{2 m+1}}-1\right)\left(\frac{V_{0}}{V}\right)^{\frac{2}{2 m+1}}\right]^{\frac{2 m-1}{2}}
$$

Now define some reduced parameters, like

$$
\epsilon=\frac{\rho}{\rho_{0}}, \eta=\frac{p}{p_{0}}, \gamma=\frac{1}{\rho_{0}^{\frac{2}{2 m+1}}}, v=\frac{V}{V_{0}}, \tau=\frac{T}{T_{0}}, \tau_{*}=\frac{T}{T_{*}}
$$

Equation (58) can be written as

$$
p=-\left[1-\left(\frac{\tau}{\tau_{*}}\right)^{\frac{2}{1-2 m}}\right]^{\frac{1-2 m}{2}}
$$


Therefore, using (59), the equations (57) and (58) become,

$$
\epsilon=\left[\gamma+\frac{1-\gamma}{v^{\frac{2}{2 m+1}}}\right]^{\frac{2 m+1}{2}}
$$

and

$$
\eta=\left[\gamma+\frac{1-\gamma}{v^{\frac{2}{2 m+1}}}\right]^{\frac{2 m-1}{2}}
$$

Also from (59), (61) and (62), we see that at $\rho=\rho_{0}, p=p_{0}, V=V_{0}, T=T_{0}$ :

$$
\epsilon=1, \eta=1, \tau=1, v=1, p_{0}=-\gamma^{\frac{1-2 m}{2}}, \tau_{*}=\gamma^{\frac{1-2 m}{2}}
$$

For example, the maximum temperature of the fluid as $T_{*}=10^{32} \mathrm{~K}$, the temperature of the Planck era, and the temperature of the tachyonic fluid at the present epoch, $T_{0}=2.7 \mathrm{~K}$ [13], then we obtain the value of $\gamma$ as $\gamma \approx 10^{\frac{64}{1-2 m}}$. If we choose $m=1 / 3$, we get $\gamma \approx 10^{192}$.

\section{DISCUSSIONS}

In this work, we have considered the general thermodynamical description for dark energy - modified Chaplygin gas and tachyonic field models in flat FRW universe. The thermal quantities i.e., pressure, density

etc. have been described in either functions of volume or temperature. For adiabatic case with $\alpha=1$, the thermal equation of state have been found and modified Chaplygin gas cools down during expansion of the universe. For tachyonic field model we have use the solution of our previous work [12] and find the corresponding equation of state. For tachyonic field model, we have used the adiabatic and thermal equation of state [13]. For adiabatic equation of state, we find the thermal quantities in terms of volume $V$ and also for thermal equation of state, the thermal quantities have been found in terms of temperature $T$. In both modified Chaplygin gas and tachyonic field models, the dark energy temperature decreases from $T^{*}$ to zero for the expansion of the universe. The entropy $S$ has been calculated in terms of $T$. It has been found that there is no critical points for these dark energy models. For thermal equation of state, we show that pressure $p$ depends on $T$ for any volume $V$. The heat capacity and speed of sound have been found in terms of volume $V$ and temperature $T$. Here, we have shown that $\left(\frac{\partial p}{\partial V}\right)_{S}<0,\left(\frac{\partial p}{\partial V}\right)_{T}=0$ and $C_{V}>0$. So from these conditions, we conclude that tachyonic field has a thermodynamically stable behaviour throughout the expansion. The determination of $T_{*}$ due to expansion for the tachyonic field have been discussed by assuming some initial conditions. Here, the thermal quantities have been investigated using some reduced parameters.

\section{Acknowledgement:}

The authors are thankful to IUCAA, Pune, India for warm hospitality where part of the work was carried out.

\section{References:}

[1] S. Perlmutter et al, Nature 39151 (1998); S. Perlmutter et al, Astrophys. J. 517565 (1999).

[2] P. M. Garnavich et al, Astrophys. J. 493 L53 (1998); A. G. Riess et al, Astron. J. 1161009 (1998).

[3] S. Bridle, O. Lahav, J. P. Ostriker and P. J. Steinhardt, Science 2991532 (2003); C. Bennett et al, Astrophys. J. Suppl. 1481 (2003); D. N. Spergel et al, Astrophys. J. Suppl. 148175 (2003);

[4] B. Ratra and P. J. E. Peebles, Phys. Rev. D 373406 (1988).

[5] R. R. Caldwell, R. Dave and P. J. Steinhardt, Phys. Rev. Lett. 801582 (1998).

[6] P. J. Steinhardt, L. Wang and I. Zlatev,Phys. Rev. Lett., 59123504 (1999).

[7] I. Zlatev, L. Wang and P. J. Steinhardt, Phys. Rev. Lett. 82896 (1999).

[8] V. Sahni and A. A. Starobinsky, Int. J. Mod. Phys. 9373 (2003); T. Padmanabhan, Phys. Rept. 380235 (2003).

[9] A. Sen, JHEP 0207065 (2002); F. Piazza and S. Tsujikawa, JCAP 0407004 (2004); Z. K. Guo, Y. S. 
Piao, X. M. Zhang and Y. Z. Zhang, Phys. Lett. B 608177 (2005); V. Sahni and Y. Shtanov, JCAP 0311 014 (2003); A. Y. Kamenshchik, U. Moschella and V. Pasquier, Phys. Lett. B 511265 (2001).

[10] Y. S. Myung, arXiv:0812.0618v1 [gr-qc].

[11] H. B. Benaoum, hep-th/0205140; U. Debnath, A. Banerjee and S. Chakraborty, Class. Quantum Grav. 21 5609 (2004); T. Bandyopadhyay and S. Chakraborty, Mod. Phys. Lett. A 242377 (2009).

[12] S. Chattopadhyay, U. Debnath and G. Chattopadhyay, Astrophys. Space Sci. 31441 (2008).

[13] F.C. Santos, M. L. Bedran and V. Soares Phys. Lett. B 63686 (2006); Phys. Lett. B 646215.

[14] L. D. Landau and E. M. Lifschitz, Statistical Physics, third ed., Course of Theoretical Physics, Vol. 5, Butterworth-Heinemann, London, 1984. 